\title{
Methods for Predicting Spectral Response of Fibers Blends
}

\author{
Rocco Furferi $\left.{ }^{(}\right)$, Lapo Governi, and Yary Volpe \\ Department of Industrial Engineering of Florence, University of Florence, \\ Via di Santa Marta 3, 50139 Firenze, Italy \\ \{rocco.furferi, lapo.governi, yary.volpe\}@unifi.it \\ http://ww. dief .unifi.it
}

\begin{abstract}
Textile companies usually manufacture fabrics using a mix of pre-colored fibers according to a traditional recipe based on their own experience. Unfortunately, mainly due to the fibers dyeing process, the colorimetric distance between the obtained fabric and the desired one results unsatisfactory with respect to a colorimetric threshold established by the technicians. In such cases, colorists are required to slightly change the original recipe in order to reduce the colorimetric distance. This trial and error process is time-consuming and requires the work of highly skilled operators. Computer-based color recipe assessment methods have been proposed so far in scientific literature to address this issue. Unlikely, many methods are still far to be reliably predictive when the fabric is composed by a high number of components. Accordingly, the present work proposes two alternative methods based on Kubelka-Munk and subtractive mixing able to perform a reliable prediction of the spectrophotometric response of a fabric obtained by means of any variation of a recipe. The assessment performed on a prototypal implementation of the two methods demonstrates that they are suitable for reliable prediction of fabric blends spectral response.
\end{abstract}

Keywords: Computer-based color assessment - Kubelka-Munk theory • Spectro-photometer $\cdot$ Fabric blend

\section{Introduction}

One of the key phases in producing textile fabrics of a desired color is the so called "recipe-based mixing" i.e. the process of mixing together a number of differently colored fibers in different percentages stated by textile companies on the basis of their know-how. Unfortunately, whichever the dyeing process, the result obtained by mixing the fibers may be very different, in terms of color, from the reference, even using the same consolidated recipe. In most cases, color differences between the recipe-based reflectance factors and the spectrophotometric response of the reference, in terms of CIELAB and $\mathrm{CMC}(2: 1)$ distances [1] under several standard illuminants [2], may be higher than 0.8 when measured using a spectrophotometer. As a consequence, companies are required to slightly change

(C) Springer International Publishing Switzerland 2015

V. Murino et al. (Eds.): ICIAP 2015 Workshops, LNCS 9281, pp. 79-86, 2015.

DOI: $10.1007 / 978-3-319-23222-5 \_10$ 
the original recipe on the basis of their experience and to produce several samples in order to reduce the gap between the color of the final product and the desired one. This trial and error approach constitutes a bottleneck of the entire fabric production process since each trial is usually performed in, at least, 40 minutes. To speed-up this process several computer-based approaches have been proposed in literature dealing with the assessment of the color matching of dyed fibers. Most of them are based on the Kubelka-Munk (K-M) theory [3,4] that is widely used for predicting the spectral reflectance for a mixture of components (colorants) that have been characterized by absorption $\mathrm{K}$ and scattering $\mathrm{S}$ coefficients. Unfortunately, the Kubelka-Munk function leads to problems in practical use due to the fact that fiber blends are not obtained by adding colorants on a substrate but, as already stated, by mixing together fibers $[5,6]$. To overcome these limitations, several studies, related to the tristimulus-matching algorithm based on the Stearns-Noechel (S-N) model [7] (and its implementations [8-11]), have been proposed so far. However S-N model provides reliable results only for blends composed by maximum $5-6$ differently colored fibers thus limiting the approach to a lower number of cases since most companies mix together up to $15-20$ elements. As a consequence, further experimentations and studies have been carried out with the aim of integrating traditional theories with more practical methods. In fact, since textile companies always create a first-attempt fabric using their recipe it is possible to have, as an additional information, the actual spectrophotometric response of at least one blend (whose reflectance values are often "near" to the reference ones). This allows to introduce, by a way of example, both theoretical approaches (using comparison between expected and actual blend reflectance values [12]), and non-deterministic models such as Artificial Neural Networks (ANNs)[13]. On the basis of considerations presented above, the present work aims to propose two alternative methods able to perform a reliable prediction of the spectrophotometric response of a fabric obtained by means of any variation of an original formula without the need of implementing trained systems such as ANNs.

\section{Methods}

\subsection{Statement of the Problem}

Let $\mathbf{p}_{i}(\lambda)(i=1,2, n)$ be the spectral reflectance factors of the $i^{\text {th }}$ component of a fabric (being $n$ the total number of components and $\lambda$ indicates the wavelength). Now, let $\mathbf{R}_{F}(\lambda)$ be the spectral reflectance factors of the fabric obtained by mixing the components $\mathbf{p}_{i}(\lambda)$ according to a given recipe $A=\left[\alpha_{1}, \alpha_{2}, \ldots, \alpha_{n}\right]$ with $\sum_{i=1}^{n} \alpha=1$. The general relationship between $\mathbf{R}_{F}(\lambda)$ and the vectors $\mathbf{p}_{i}(\lambda)$ may be stated by the following formula:

$$
\mathbf{R}_{F}(\lambda)=\mathcal{F}\left(A, \mathbf{p}_{i}(\lambda)\right)
$$

Varying the wavelength in the range [400 $700 \mathrm{~nm}$ ] and with a $10 \mathrm{~nm}$ step, the size of vectors $\mathbf{p}_{i}(\lambda)$ and $\mathbf{R}\left(\lambda, \alpha_{i}\right)$ is $1 \times 31$. According to Eq. 1, if a method 
for evaluating the transfer function $\mathcal{F}$ is established, it is possible to evaluate the spectral reflectance factors of a fabric $\mathbf{R}_{F}(\lambda)$ given the parameters $\alpha_{i}$ and the vectors $\mathbf{p}_{i}(\lambda)$. As mentioned in the introductory section, the determination of the transfer function is not straightforward, especially for blends composed by more than $5-6$ components (i.e. for $n>6$ ) when theoretical approached such as K-M and S-N have been demonstrated to provide inaccurate results.

\subsection{K-M-Based Approach}

K-M theory states a correlation between the K-S ratio of a blend ( $\mathrm{mix}$ ) and the K-S ratio of singular components to be mixed together plus the substrate according to the following equation:

$$
\left(\frac{K}{S}\right)_{\lambda, m i x}=\left(\frac{k_{\lambda, t}}{s_{\lambda, t}}\right)+\alpha_{1}\left(\frac{k_{\lambda, 1}}{s_{\lambda, 1}}\right)+\ldots+\alpha_{n}\left(\frac{k_{\lambda, n}}{s_{\lambda, n}}\right)
$$

Where the term $\Psi_{S}(\lambda)=\left(\frac{k_{\lambda, t}}{s_{\lambda, t}}\right)$ is the ratio between absorption and scattering of the substrate. In blends obtained by mixing fibers, the definition of "substrate" is quite weak since unlike fabrics dipped in dye bath (where a "monochrome" substrate is dyed) the final product is obtained using pre-colored fibers. As a consequence the scattering and absorption coefficients of the substrate are not evaluable. For this reason the K-M approach is not suitable for predicting the color of such blends. Fortunately, since colorists working in textile companies always create a first-attempt blend using their historical recipe, the actual reflectance factors of the blend are known together with the recipe and the spectra of each component. This additional information may be used to evaluate a K-S ratio $\boldsymbol{\Psi}_{S}^{*}(\lambda)$ by using Eq. 2.

$$
\boldsymbol{\Psi}_{S}^{*}(\lambda)=\left(\frac{K}{S}\right)_{\lambda, m i x}-\left\{\alpha_{1}\left(\frac{k_{\lambda, 1}}{s_{\lambda, 1}}\right)+\ldots+\alpha_{n}\left(\frac{k_{\lambda, n}}{s_{\lambda, n}}\right)\right\}=\Psi_{F}(\lambda)-\Psi_{C}(\lambda)
$$

Even if $\boldsymbol{\Psi}_{S}^{*}(\lambda)$, in the present work named "K-S ratio of an equivalent fabric substrate", has not a physical meaning, it describes the K-M ratio of an ideal equivalent fabric having the same reflectance values of the actual ones but obtained using a dye dipping process. Since, as already mentioned, such a term is defined in a theoretical way, and no physical counterpart actually exists, it may consist also of negative values in the 400-700 nm wavelength range. In Fig. 1 the terms $\boldsymbol{\Psi}_{F}, \boldsymbol{\Psi}_{S}^{*}$ and $\boldsymbol{\Psi}_{C}$ of Eq. 3 for 4 fabric samples are shown.

Under the hypothesis that the turbid mixing mechanism of fibers only slightly changes by varying the original recipe (i.e. by changing the values $\alpha_{i}$ ), it can be assumed that $\boldsymbol{\Psi}_{S}^{*}$ is constant for a given fabric. As a consequence the K-S ratio for any given variation of recipe $\boldsymbol{\Psi}_{\text {Fnew }}$ can be evaluated as follows:

$$
\boldsymbol{\Psi}_{\text {Fnew }}=\boldsymbol{\Psi}_{S}^{*}(\lambda)+\overline{\boldsymbol{\Psi}}_{C}(\lambda)
$$




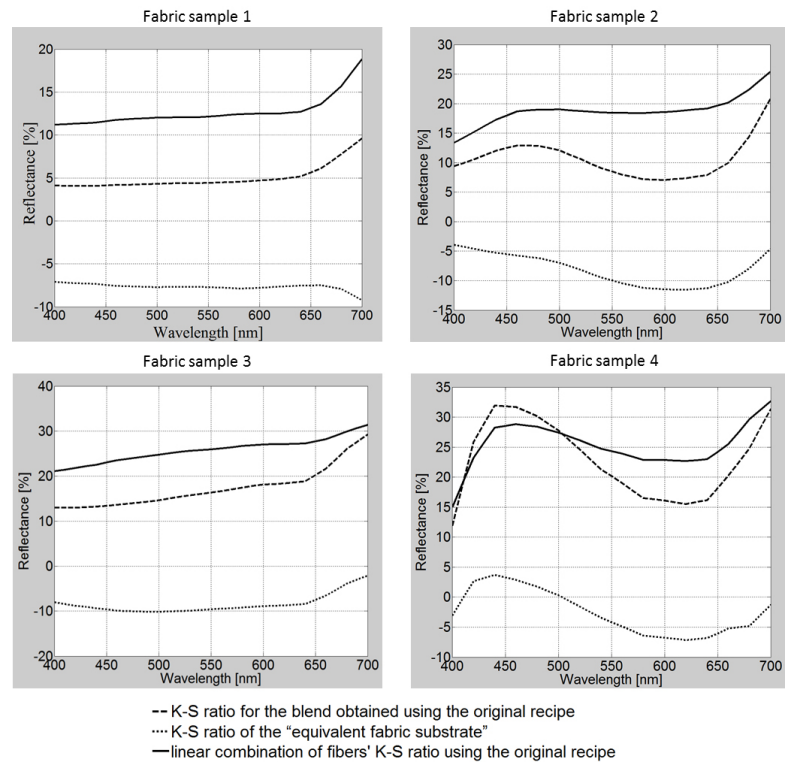

Fig. 1. Terms $\boldsymbol{\Psi}_{F}, \boldsymbol{\Psi}_{S}^{*}$ and $\boldsymbol{\Psi}_{C}$ of Eq. 3 for 4 fabric samples

where $\overline{\mathbf{\Psi}}_{C}(\lambda)$ is the linear combination of fibers' K-S ratio using the modified recipe. Once $\boldsymbol{\Psi}_{\text {Fnew }}$ is calculated using Eq. 4, it is straightforward to evaluate the spectral response $\mathbf{R}_{F}^{*}(\lambda)$, constituting the best prediction of actual $\mathbf{R}_{F}(\lambda)$, by using the following equation [5]:

$$
\boldsymbol{\Psi}_{\text {Fnew }}(\lambda)=\frac{\left(1-\mathbf{R}_{F}^{*}(\lambda)\right)^{2}}{2 \mathbf{R}_{F}^{*}(\lambda)}
$$

The correctness of Eq. 5 is validated in Section 3.

\subsection{Subtractive Mixing-Based Approach}

As widely known, in case pigments are mixed together, the subtractive color mixing model is reliable in predicting the spectrophotometric response of the blend [14]. The density of the pigments $\mathbf{d}_{i}(\lambda)$ (for any given wavelength) are approximately additive and can be expressed as follows:

$$
\mathbf{d}_{i}(\lambda)=-\log _{10}\left(\mathbf{p}_{i}(\lambda)\right)
$$

Accordingly, it is possible to define a subtractive color mixing spectrum $\mathbf{R}_{S}\left(\lambda, \alpha_{i}\right)$ as:

$$
\mathbf{R}_{S}\left(\lambda, \alpha_{i}\right)=\exp \left(\sum_{i=1}^{n} \alpha_{i} \mathbf{d}_{i}(\lambda)\right)
$$


As stated in the scientific literature, and further demonstrated in the industrial practice, Eq. 7 is not appropriate for the evaluation of the spectral reflectance factors of fabrics. Actually, unlike - for instance - mixtures of paints, it is not possible to obtain a complete homogenization of textile fibers because they remain separate entities on a macroscopic scale. The difference between reflectance values $\mathbf{R}_{S}\left(\lambda, \alpha_{i}\right)$ and $\mathbf{R}_{F}(\lambda)$ for 4 fabrics with different compositions are depicted in Fig. 2, showing that the color subtractive model is not suitable as it stands for predicting the fabric reflectance values.

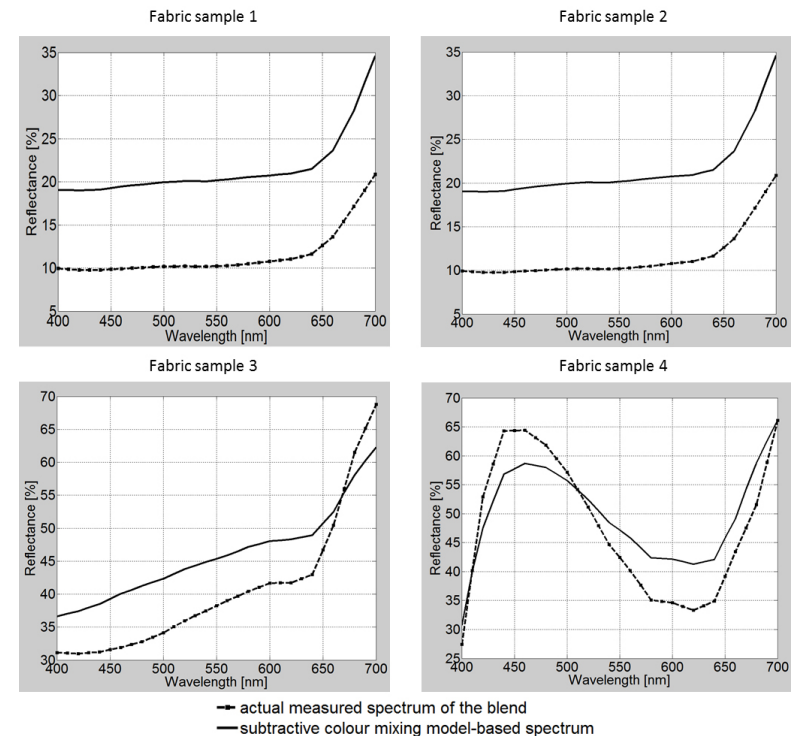

Fig. 2. Difference between actual measured spectrum and subtractive color mixing model-based estimation for the 4 fabric blends of Fig. 1

However, the definition of $\mathbf{R}_{S}\left(\lambda, \alpha_{i}\right)$ can be considered a very rough approximation of the real blend spectrum, to be corrected using an appropriate wavelength-dependant function $\boldsymbol{\Phi}(\lambda)$ in order to evaluate a predicted $\mathbf{R}_{F}^{* *}(\lambda)$ of $\mathbf{R}_{F}(\lambda)$ as follows:

$$
\mathbf{R}_{F}^{* *}(\lambda)=\mathbf{\Phi}(\lambda) \cdot \mathbf{R}_{S}\left(\lambda, \alpha_{i}\right)
$$

Since, as already stated, in the industrial practice a first-attempt fabric is physically realized using the standard recipe, it is possible to know for such a blend both the vectors $\mathbf{R}_{F}(\lambda)$ - measured using a spectrophotometer - and $\mathbf{R}_{S}\left(\lambda, \alpha_{i}\right)$. This additional information allows the determination of the transfer function $\boldsymbol{\Phi}(\lambda)$ as follows:

$$
\boldsymbol{\Phi}(\lambda)=\frac{\mathbf{R}_{F}(\lambda)}{\mathbf{R}_{S}\left(\lambda, \alpha_{i}\right)}
$$


Under the hypothesis that the transfer function $\boldsymbol{\Phi}(\lambda)$ remains unchanged once the recipe is changed, the spectral response $\mathbf{R}_{F n e w}^{* *}(\lambda)$ of a fabric obtained by changing the original recipe is provided by:

$$
\mathbf{R}_{\text {Fnew }}^{* *}(\lambda)=\frac{\mathbf{R}_{F}(\lambda)}{\mathbf{R}_{S}\left(\lambda, \alpha_{i}\right)} \cdot \mathbf{R}_{\text {Snew }}\left(\lambda, \alpha_{i}\right)
$$

Where $\mathbf{R}_{\text {Snew }}\left(\lambda, \alpha_{i}\right)$ is the subtractive colour mixing spectrum obtained by changing the weights $\alpha_{i}$ according to the new recipe and the $\mathbf{R}_{F n e w}^{* *}(\lambda)$ is the best prediction of actual $\mathbf{R}_{F}(\lambda)$ for modified recipe. The correctness of the Eq. 10 is validated in Section 3.

\section{Results}

The two prediction methods described above have been validated thanks to the collaboration of the staff of the Textile Company New Mill s.p.a. - Prato (Italy) by using a set of 80 fabrics obtained adopting their standard recipe (40 fabrics used to evaluate $\boldsymbol{\Psi}_{S}^{*}(\lambda)$ and $\boldsymbol{\Phi}(\lambda)$ ) and a modified version (40 fabrics to evaluate the two methods). Both the raw materials and the blends obtained by mixing them were processed by using an acquisition system consisting of a bench on which a Hunterlab Ultrascan VIS reflectance spectrophotometer is placed and connected to a PC. The final results of this experimental setup are: the spectral responses of the 40 samples obtained using the standard recipe; the 40 spectral responses for the modified recipe and the spectra of each raw material composing the blends. The predicted spectra $\left(\mathbf{R}_{F}(\lambda)\right.$ and $\left.\mathbf{R}_{F n e w}^{* *}(\lambda)\right)$ obtained using the two proposed approaches are then compared in terms of $\mathrm{CMC}(2: 1)$ distance with the actual measurement of the real fabrics obtained using the modified recipes. Moreover a comparison between the results obtained using the two approaches provided by [12] and [13] is proposed. In Table 1 the aggregate results of the comparison for the 40 samples are shown.

In Fig. 3 the predicted spectra obtained using respectively the K-M-based approach, the Subtractive mixing-based approach, the theoretical method provided in [12] and the ANN-based method provided in [13] are plotted for the 4 exemplificative samples.

Table 1. Aggregate results obtained by comparing the two proposed methods with tho once described in [12] and [13]

\begin{tabular}{|l|c|c|c|c|}
\cline { 2 - 5 } \multicolumn{1}{c|}{} & \multicolumn{4}{c|}{ CMC(2:1) distance from reference } \\
\cline { 2 - 5 } & $\begin{array}{c}\text { K-M-based } \\
\text { approach }\end{array}$ & $\begin{array}{c}\text { Subtractive } \\
\text { mixing-based } \\
\text { approach }\end{array}$ & $\begin{array}{c}\text { Theoretical } \\
\text { approach [12] }\end{array}$ & $\begin{array}{c}\text { ANN-based } \\
\text { approach [13] }\end{array}$ \\
\hline Mean value & 0.5633 & 0.5260 & 0.7886 & 0.4761 \\
Median value & 0.5080 & 0.4738 & 0.7589 & 0.4438 \\
Max value & 1.0924 & 1.0280 & 1.4272 & 0.9982 \\
Min value & 0.1283 & 0.1285 & 0.4231 & 0.1058 \\
Variance & 0.047 & 0.045 & 0.054 & 0.041 \\
\hline
\end{tabular}



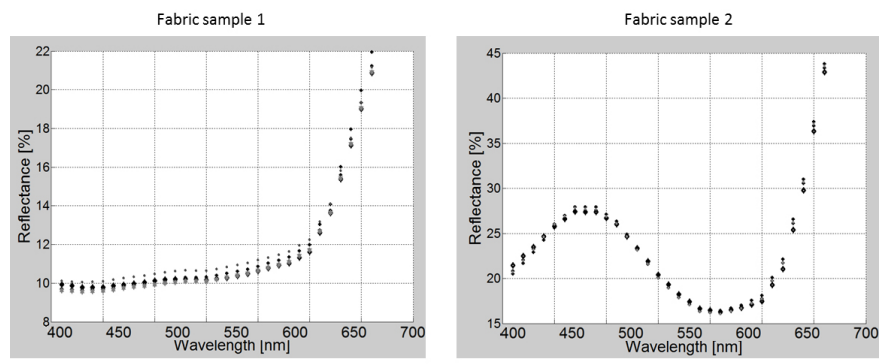

Fabric sample 3
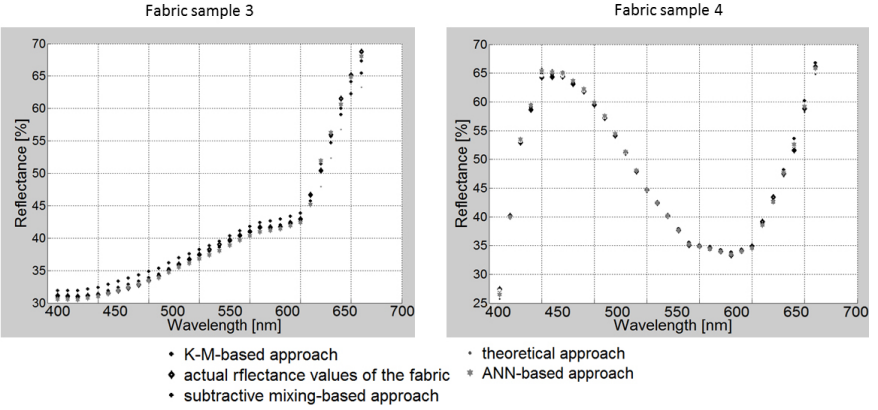

Fig. 3. Predicted spectra obtained using respectively the K-M-based approach, the Subtractive mixing-based approach, the theoretical method provided in [12] and the ANN-based method provided in [13]

\section{Conclusions}

The present paper described two methods for predicting the spectral response of a fabric obtained by mixing pre-colored fibers. The first method implements a simplified version of the Kubelka-Munk two constant approach by introducing a term named "K-S ratio of an equivalent fabric substrate" used to provide a robust prediction once a first-attempt fabric (blend) is physically realized. The second method uses a rough prediction based on a subtractive color mixing model to build a transfer function between the spectral responses of the raw materials composing the blend and the expected reflectance factors of the blend. Results of validation test show that, as expected, the ANN-based method is the one that generally provides better results in predicting the actual color of the blend (average $\mathrm{CMC}(2: 1)$ distance less than 0.48) but the two proposed methods perform well with a color distance averagely equal to 0.5633 and 0.5260 respectively for K-M-based and subtractive mixing-based methods. This is a noticeable result since the two proposed methods does not requires training phases as is the case for ANN-based ones. The prediction errors, i.e. the color distance, increases for blends characterized by high number of raw materials (i.e. ¿16). However, a prediction error lower than 1.2 for such cases has been measured; this is acceptable for many companies. Both in terms of mean and median values, subtractive mixing-based method performs slightly better than 
the K-M-based ones; both methods, in any case, provide a closer prediction when compared with theoretical approach proposed in [12]. Future work will be addressed to increase the validation set and to further increase the performance especially for recipes characterized by a high number of differently colored fibers and in assessing the influence of the roughness of fibers composing the blend.

\section{References}

1. Aspland, R., Shanbhag, P.: Comparison of color difference equations for textiles. CMC (2: 1) and CIEDE2000. AATCC review 4(6), 26-30 (2004)

2. Furferi, R., Governi, L., Volpe, Y.: Image processing-based method for glass tiles color matching. Imaging Science Journal 61(2), 183-194 (2013)

3. Amirshahi, S.H., Pailthorpe, M.T.: Applying the Kubelka-Munk equation to explain the color of blends prepared from precolored fibers. Textile research journal 64(6), 357-364 (1994)

4. Amirshahi, S.H., Pailthorpe, M.T.: An algorithm for the optimization of color prediction in blends. Textile Research Journal 65(11), 632-637 (1995)

5. Burlone, D.A.: Effect of Fibre Translucency on the Color of Blends of Precolored Fibres. Textile Research Journal 60(3), 162-167 (1990)

6. Hongying, Y., Zhu, S., Pan, N.: On the Kubelka-Munk Single-Constant/TwoConstant Theory. Textile Research Journal 80(3), 263-270 (2010)

7. Steams, E.I., Noechel, F.: Spectrophotometric Prediction of Color Wool Blends. Am. Dyest 33(9), 177-180 (1944)

8. Rong, L.I., Feng, G.U.: Tristimulus algorithm of colour matching for precoloured fibre blends based on the Stearns- Noechel model. Coloration Technology 122(2), 74-81 (2006)

9. Thompson, B., Hammersley, M.J.: Prediction of the colour of scoured-wool blends. Journal of the Textile Institute 69(1), 1-7 (1978)

10. Kazmi, S.Z., Grady, P.L., Mock, G.N., Hodge, G.L.: On-line color monitoring in continuous textile dyeing. ISA Transactions 35(1), 33-43 (1996)

11. Philips-Invernizzi, B., Dupont, D., Jolly-Desodt, A.M., Caze, C.: Color formulation by fiber blending using the Stearns -oechel model. Color Research and Application 27(2), 100-107 (2002)

12. Furferi, R., Carfagni, M.: Colour mixing modelling and simulation: Optimization of colour recipe for carded fibres. Modelling and Simulation in Engineering, vol. 2010, Article ID 487678, 9 p. (2010)

13. Furferi, R., Governi, L.: Prediction of the spectrophotometric response of a carded fiber composed by different kinds of coloured raw materials: An artificial neural network-based approach. Color Research and Application 36(3), 179-191 (2011)

14. Hawkyard, C.J.: Synthetic reflectance curves by subtractive colour mixing. Journal of the Society of Dyers and Colourists 109(78), 246-251 (1993) 